\title{
Dentofacial characteristics of oral breathers in different ages: a retrospective case-control study
}

\author{
Rosa Carrieri Rossi ${ }^{*}$, Nelson José Rossi ${ }^{2}$, Nelson José Carrieri Rossi ${ }^{2}$, Hélio Kiitiro Yamashita ${ }^{3}$ \\ and Shirley Shizue Nagata Pignatari ${ }^{4}$
}

\begin{abstract}
Background: This study aimed to investigate the dental and skeletal variables associated with disturbances of craniofacial development in oral-breathing $(\mathrm{OB})$ individuals and the probability that these variables are related to this condition.

Methods: This is an observational retrospective case-control study of 1596 patients divided into three groups of age n1 5-12, n2 13-18, and n3 19-57 years. Radiographic, clinical, and models data were analyzed. The control group was consisted of nasal breathing (NB) individuals. Statistical analyses of the qualitative data were performed with $x^{2}$ test to identify associations, and odds ratio (OR) tests were performed for the variables that the chi-square test $\left(x^{2}\right)$ identified an association.
\end{abstract}

Results: In the descriptive analysis of the data, we observed that the class II malocclusion was the most frequent in the total sample, but when divided by age group and mode of breathing, there is a random division of these variables. In $n 1$ group, class II, $(\mathrm{OR}=2.02)$ short and retruded mandible ( $\mathrm{SM}$ and $\mathrm{RM})(\mathrm{OR}=1.65$ and 1.89$)$ were associated with $\mathrm{OB}$ and it was considered a risk factor. In $\mathrm{n} 2$ group, class $I I(O R=1.73), \mathrm{SM}(\mathrm{OR}=1.87)$ and increased lower anterior height (ILAFH) $(\mathrm{OR}=1.84)$ seemed to be associated and to be risk factors for OB. In the $n 1$ group, decreased lower anterior facial height (DLAFH) and brachycephalic facial pattern (BP) seemed to be associated with NB and a protective factor against oral breathing.

Conclusions: This study showed that dental and skeletal factors are associated with OB in children, and it seems that it becomes more severe until adolescence. But adults showed no associations between OB and skeletal factors, only in dental variables, indicating that there is no cause-effect relationship between the dental and skeletal factors and OB. The treatment of nose breathing patient should be multidisciplinary, since OB remains even when dental and skeletal factors slow down.

Keywords: Mouth breathing; Oral breathing; Respiration

\section{Background}

Although the importance of nasal breathing (NB) for craniofacial growth and harmonious development is well established, a large number of studies also address oral breathing $(\mathrm{OB})$ and its role in craniofacial changes [1]. The difficulty in understanding the causes and effects of respiratory patterns on craniofacial growth is a reflex of the fact that several factors acting simultaneously can influence it, and there is often inaccuracy in the diagnosis of oral and nasal breathing [2-25].

\footnotetext{
* Correspondence: rosacrossi@gmail.com

'Division of Pediatric Otolaryngology, Federal University of Sao PauloUNIFESP Brasil, Rua Botucatu 740, 4 andar, V. Clementino, São Paulo CEP:04023-062, Brazil

Full list of author information is available at the end of the article
}

Some authors have claimed that $\mathrm{OB}$ alters growth and facial development [1, 2, 4, 7, 10-13, 16, 19, 21, 22, 24, 25], but others disagree and believe that the altered growth of the dentofacial complex results from other factors that may predispose individuals to $\mathrm{OB}[2-7]$.

The literature has not yet clearly demonstrated the relationship between $\mathrm{OB}$ and the growth and development. Addressing these questions is important since patients with such respiratory disturbances more frequently exhibit chronic sleep apnea and higher mortality rates and are more likely to develop cardiovascular diseases [7].

This study aimed to investigate the dental and skeletal variables associated with disturbances of craniofacial 
development in oral breathing individuals and the probability that these variables are related to this condition.

\section{Methods}

This observational retrospective case-control study was approved by the Ethics Committee of the Federal University of São Paulo, protocol number 103.275. Informed consent was obtained from all patients or legal guardians.

The study population $(N=1596)$ was based on a consecutive sample of oral and nose breathing patients of both sexes between ages 5 and 57 years who sought orthodontic treatment in an orthodontics clinic in the city of São Paulo between the years 2006 and 2012.

The sample was consecutive and consisted of a large number of subjects in an attempt to control errors through descriptive and inferential statistical tests. The survey was conducted by the same examiner in a uniform manner to ensure consistency and quality of results [1, 2, 8-11].

Initially, the 1596 patients $(N)$ were divided in three groups according to age range $(\mathrm{n} 1, \mathrm{n} 2, \mathrm{n} 3)$, as previous studies:

$$
\begin{aligned}
& \text { n1- } 5 \text { to } 12 \text { years old }(n=523) \text { child }^{1,6,8,10,12,20} \\
& \text { n2- } 13 \text { to } 18 \text { years old }(n=443) \text { adolescent }{ }^{2,4,5,7,21} \\
& \text { n3- } 19 \text { to } 57 \text { years old }(n=340) \text { adult }
\end{aligned}
$$

All patients had previous examinations of the same diagnostic center consisting of plaster model and side and panoramic radiographs with McNamara's cephalometric analysis [24]. Measures such as the size of the mandible (NM, RM, PM), the lower anterior facial height (NLAFH, ILAFH, DLAFH), the position of the maxilla (NMx, RMx, $\mathrm{PMx}$ ), and mandible (NM, LM, SM), and facial pattern (NP, BP, DP) were evaluated [24, 25].

Dental characteristics were obtained by prior clinical examination and model analysis. The position of the first permanent molars followed the Angle classification [26]. Intermolar (IM) and intercanine (IC) widths (W), superior (S) and inferior (I) widths were obtained in orthodontic model with a digital caliper measuring the distance between the central fosse of molars and between the cusp tip of the canines $[5,6,19]$.

The patients were divided by type of breathing presented, using the protocol described in previous studies [11]. According to their predominant breathing pattern history and the findings on clinical examination, patients were classified as either $\mathrm{OB}$ or NB. The $\mathrm{OB}$ was considered as a study group (SG) and the NB as a control group (CG).

The orthodontist performed the history and clinical examination including evaluation of lips protrusion or retrusion, and the otolaryngologist was responsible for the speech and audiologic evaluations. Patients and their guardians answered a questionnaire concerning the breathing habits, while awake, with respect to any permanency of breathing with the mouth open, oral breathing, nasal obstruction, oral malodor, hyponasal speech, and while asleep, considering snoring, sleep apnea [7], restless sleep, or hyper salivation. Most of the questions were yes or no items as the protocol [11].

Patients who reported harmful habits such as finger sucking, the use of a pacifier during sleep or during the day, patients who had been submitted to orthodontic treatment, and those whose complete record could not be verified, were excluded. The data were collected in an Excel (Microsoft, 2010) database.

Comparison of OB and NB with dental and skeletal variables was accomplished among the three age groups (n1, n2, n3).

Qualitative variables were described by means of tables showing the percentage of occurrences for each category. After the division of the groups according to exclusion criteria, each variable had different $n$, in the tables. Correlation analysis was made between the pattern of breathing and the following variables:

1. breathing mode and gender

2. breathing mode and Angle class

3. breathing mode and maxilla position

4. breathing mode and mandible position

5. breathing mode and mandible size

6. breathing mode and anterior-lower facial height

7. breathing mode and facial pattern

Variables OB and NB groups, separated by age (n1, n2, n3), were evaluated by the homogeneity test (chi-square$x^{2}$ ). Pearson correlations were selected for a reliability of $95 \%$ and a significance level of $p<0.05$. Odds ratio (OR) tests were performed for the variables that the chisquare test $\left(x^{2}\right)$ identified an association.

The purpose of these tests was to identify the variable associated with $\mathrm{OB}$ and to determine the relative influence of these variables on the risk (OR) of developing the disease. When evidence of a correlation was found, we concluded that the variable was dependent of the breathing pattern.

Quantitative variables were described using measures of central tendency (mean and median) and measures of dispersion as standard deviation (SD). The Student's $t$ test was used to investigate the possible associations between the quantitative variables with confidence interval (CI) $95 \%$ in each of the three groups (n1, n2, n3). Means and SD for the $\mathrm{OB}$ and NB, and the upper and lower intermolar and intercanine widths (IMSW, IMIW, ICSW, ICIW), were checked with the Minitab program, version 14.

For rejecting the null hypothesis we fixed the significance level of $5 \%$. 


\section{Results}

In the descriptive analysis of the data, we observed that the class II malocclusion was the most frequent in the total sample, but when divided by age group and mode of breathing, there is a random division of these variables (Figs. 1 and 2).

\section{Group $1(\mathrm{n} 1=523)$ 5-12 years-Qualitative variables}

For ages 5-12 years old, there was no evidence of association between gender, class III malocclusion, protruded $(\mathrm{PMx})$ and retruded maxilla ( $\mathrm{RMx}$ ), protruded mandible (PM), large mandible (LM), dolichocephalic pattern (DP), increase in lower anterior face height (ILAFH), and the type of breathing.

There was a significant association between class II malocclusion, retruded mandible (RM) and short mandible (SM); there was a significantly higher percentage of oral breathing with these variables. A higher percentage of NB with normal mandible (NM) was also observed.

An association between decreased LAFH (DLAFH) and the type of breathing was observed, as was a higher percentage of OB with normal LAFH (NLAFH) and a higher percentage of NB with DLAFH. Finally, in the association between the brachycephalic facial pattern (BP) and the type of breathing, a higher percentage of $\mathrm{OB}$ with normocephalic pattern (NP) and a higher percentage of NB with BP were found.

We observed odds ratio (OR) between class II malocclusion $(\mathrm{OR}=2.02$; CI $(95 \%)=(1.32,3.09))$, RM $(\mathrm{OR}=1.89$; CI $(95 \%)=(0.99,3.60)), \mathrm{SM}(\mathrm{OR}=1.65$; CI $(95 \%)=(1.06$, 2.58)) with OB.

We observed DLAFH and BP with NB, and these last two presented themselves as protective factors for the development of $\mathrm{OB}(\mathrm{OR}=0.44 ; \mathrm{CI}(95 \%)=(0.26,0.77))$ and $(\mathrm{OR}=0,43, \mathrm{CI}(95 \%)=(0.24,0.78))$, respectively.

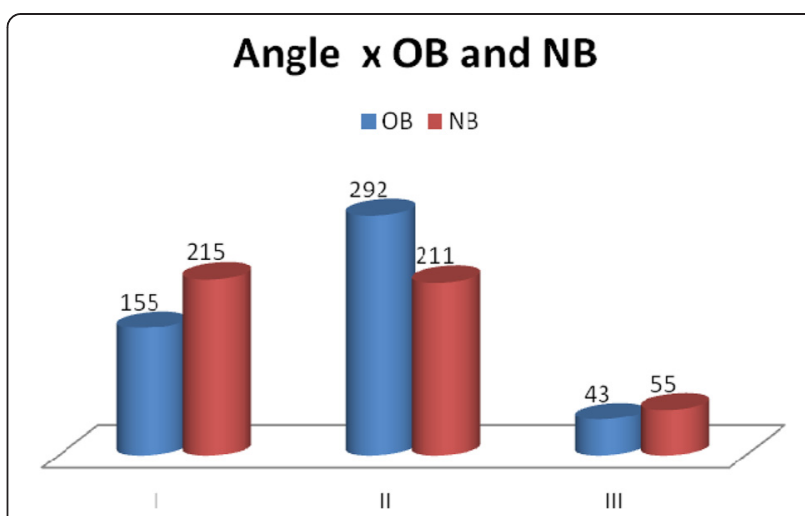

Fig. 2 Frequency of Angle classes among $O B$ and NB. There was an homogeneous distribution of Angle malocclusion classes between $\mathrm{OB}$ and $\mathrm{NB}$

The results of $x^{2}$ and Pearson associations, OR for qualitative variables tests, in group 1 are shown in Table 1 and Fig. 3.

\section{Quantitative variables}

There was no evidence of a difference between the mean ages of the $\mathrm{OB}$ and NB in group 1. No differences between the means of the upper and lower intermolars widths were detected between the OB and NB. No evidence of differences in the means of the upper and lower intercanine widths were found.

Summaries of the values of the middle range are shown in Table 2.

Group 2 ( $n 2$ = 443) 13-18 years old-qualitative variables For the age group 13-18 years old, there is no evidence of an association between gender, class III malocclusion, PMx

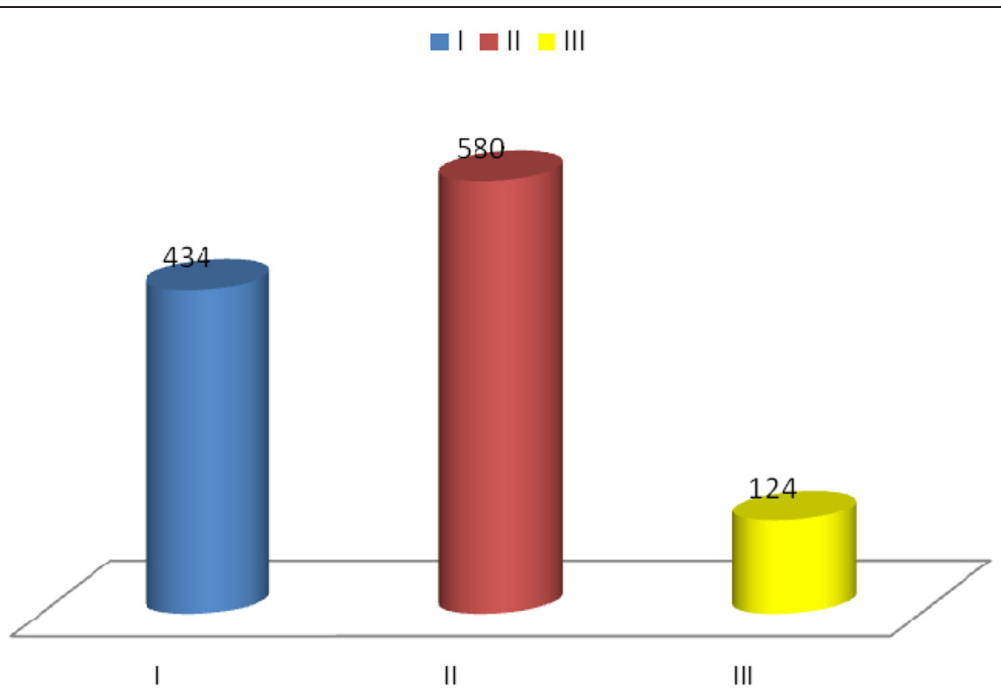

Fig. 1 Angle malocclusion in the population study. There was a greater frequency of Angle Class II in the population study 
Table 1 Intra-group association tests (Pearson's $x^{2}$, odds ratio) of the qualitative variables-group $n 1$ (5 to 12 years of age)

\begin{tabular}{|c|c|c|c|c|c|c|c|c|c|c|c|}
\hline Variables & $\mathrm{OB}(n)$ & OB \% & NB $(n)$ & NB \% & Total & Pearson $x^{2}$ & $p$ value & DF & Lilihood $x^{2}$ & OR & Cl $95 \%$ \\
\hline $\mathrm{F}$ & 152 & 54 & 130 & 63 & & 2.423 & 1.11 & 1 & 2.43 & & \\
\hline M & 125 & 46 & 80 & 38 & & & & & & & \\
\hline Total & 277 & & 210 & & 487 & & & & & & \\
\hline I & 67 & 28 & 73 & 41 & & & & & & & \\
\hline$\|$ & 156 & 64 & 84 & 48 & & 10.717 & $0.001^{*}$ & 1 & 10672 & 2.02 & (1.32:3.09) \\
\hline III & 20 & 8 & 20 & 11 & & 0.057 & 0.811 & 1 & 0.057 & & \\
\hline Total & 243 & & 177 & & 420 & & & & & & \\
\hline NMx & 25 & 9 & 17 & 8 & & & & & & & \\
\hline PMx & 181 & 66 & 146 & 71 & & 0.263 & 0.608 & 1 & 0.264 & & \\
\hline RMx & 68 & 25 & 42 & 21 & & 0.067 & 0.795 & 1 & 0.067 & & \\
\hline Total & 274 & & 205 & & 479 & & & & & & \\
\hline NM & 25 & 9 & 25 & 12 & & & & & & & \\
\hline PM & 149 & 54 & 126 & 62 & & 0.297 & 0.586 & 1 & 0.297 & & \\
\hline RM & 100 & 37 & 53 & 26 & & 3.758 & $0.053^{*}$ & 1 & 3,693 & & \\
\hline Total & 274 & & 204 & & 478 & & & & & & \\
\hline NM & 71 & 26 & 70 & 34 & & & & & & & \\
\hline IM & 85 & 32 & 65 & 32 & & 1.164 & 0.281 & 1 & 1.165 & 1.65 & $(1.06 ; 2.58)$ \\
\hline SM & 114 & 42 & 68 & 34 & & 4.898 & $0.027^{*}$ & 1 & 4.897 & & \\
\hline Total & 270 & & 203 & & 473 & & & & & & \\
\hline NLAFH & 70 & 26 & 41 & 20 & & & & & & & \\
\hline ILAFH & 168 & 62 & 115 & 57 & & 0.456 & 0.499 & 1 & 0.459 & 2.31 & $(1.28 ; 4.15)$ \\
\hline DLAFH & 34 & 12 & 46 & 23 & & 7.926 & $0.005^{*}$ & 1 & 7.957 & 0.43 & $(0.24 ; 0.78)$ \\
\hline Total & 272 & & 202 & & 474 & & & & & & \\
\hline NP & 65 & 24 & 33 & 16 & & & & & & & \\
\hline BP & 56 & 21 & 64 & 32 & & 8.442 & $0.004^{*}$ & 1 & 1.746 & 0.44 & $(1.30 ; 3.90)$ \\
\hline DP & 146 & 55 & 106 & 52 & & 1.746 & 0.186 & 1 & 1.767 & 3.25 & $(0.26 ; 0.77)$ \\
\hline Total & 267 & & 203 & & 470 & & & & & & \\
\hline
\end{tabular}

*Significant values

The values with $\left(^{*}\right)$ and in italics represent significant values

and RMx, PM and RM, LM, BP and DP, and the type of respiration

There is an association between class II malocclusion, $\mathrm{SM}$ and ILAFH, and OB. It can be seen that there is a higher percentage of $\mathrm{OB}$ with these variables and that there is a higher percentage of NB with class I malocclusion and of NB with NLAFH.

We calculated the OR for class II malocclusion $(\mathrm{OR}=1.73$; CI $(95 \%)=(1.07,2.78), \mathrm{SM}(\mathrm{OR}=1.87$; $\mathrm{CI}$ $(95 \%)=(1.07,3.24)$ ILAFH $(\mathrm{OR}=1.84$, CI $(95 \%)=$ $(1.07,3.17)$ with OB. The results of $x^{2}$ and Pearson associations, OR for qualitative variables tests, in group 2 are shown in Table 3 and Fig. 4.

\section{Quantitative variables}

There is a significant difference between the mean age of the $\mathrm{OB}$ and $\mathrm{NB}$ for the age group 13-18 years. The
OB subjects are on average 0.4 years younger than NB, with the $95 \%$ confidence level between 0.06 and 0.8 years.

There is no evidence of a difference between the average width of the maxilla and the average width of mandible, as determined by the intermolar distances and the upper and lower intercanine distances of oral and nose breathing adolescents in the 13 to 18 age group.

Summaries of the average range values are shown in Table 4.

Group 3 (n3 =312) 19-57 years old-qualitative variables For the age group 19-57 years old, there is no evidence of an association between gender, class III malocclusion, PMx and RMx, PM and RM, LM and SM, BP and DP, and mode of breathing. 


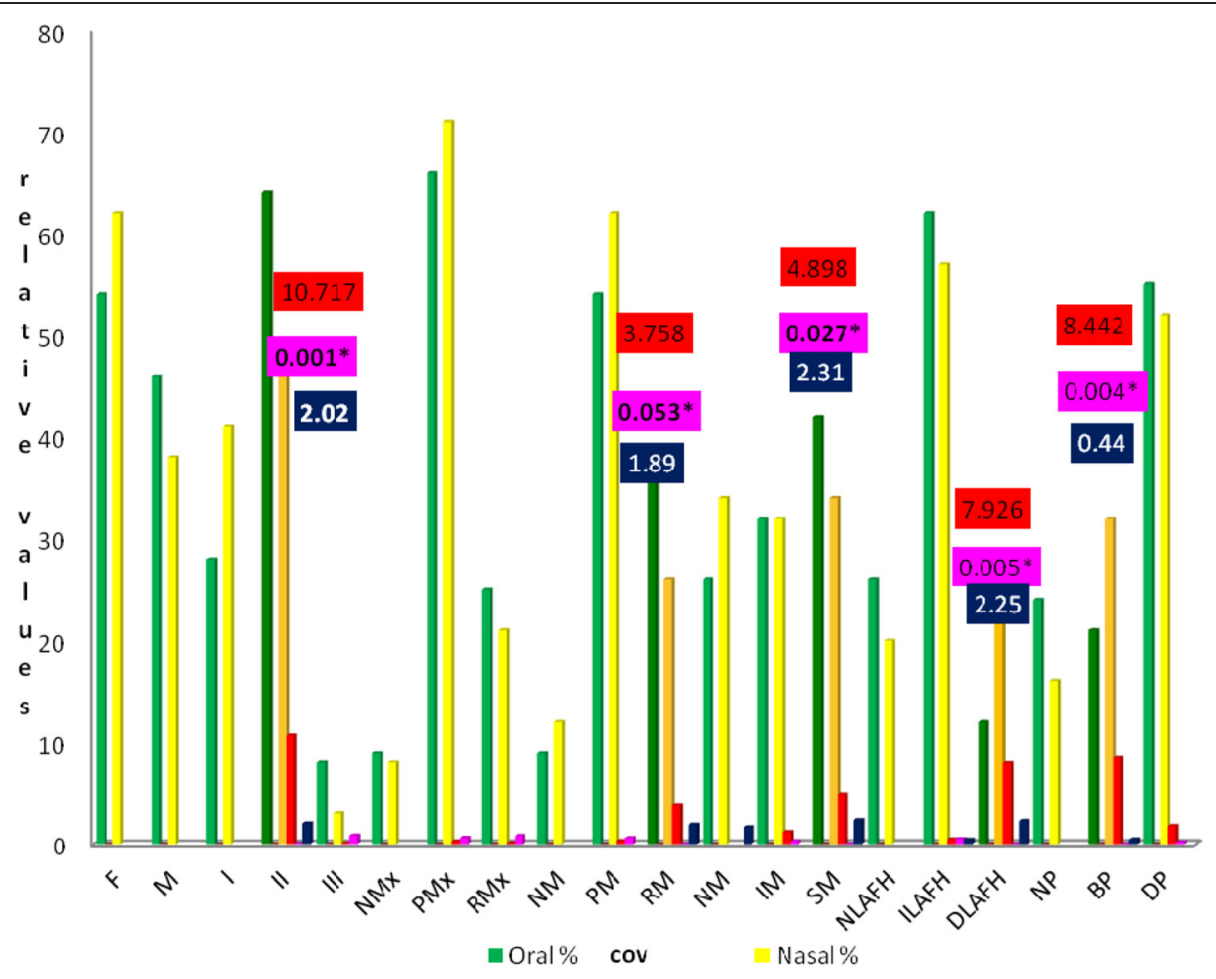

Fig. 3 Intra-groups association values (Pearson's $x^{2}$, OR) for qualitative variables, group n1 (523), 5 to 12 years of age, with OB and NB. * Significant values. The variables that showed significant associations and risk of disease were Class II, RM, SM. DLAFH and BP (protective factors)

For the age group of 19 to 57 years we found a marginally significant association between classification II malocclusion and oral breathing $(\mathrm{OR}=1.72$; $\mathrm{CI}(95 \%)=$ $(0.99,2.98))$.

The results of intra-group association tests of qualitative variables (Pearson's chi-square and odds ratio) for the age group $19-57$ years with $(\mathrm{n} 3=312)$ are shown in Table 5 and Fig. 5.

\section{Quantitative values}

There is a significant difference between the average width of the mandibles in $\mathrm{OB}$ subjects in group 3. The

Table $2 T$ tests for difference in the averages - quantitative values of $n 1$ (5 to 12 years of age)

\begin{tabular}{llllll}
\hline & $(n)$ & IMSW & IMIW & ICSW & ICIW \\
\hline OB & 277 & 67 & 64 & 54 & 57 \\
NB & 212 & 49 & 43 & 47 & 46 \\
Means OB & 9.56 & 47.04 & 45.3 & 33.76 & 27.53 \\
Means NB & 9.79 & 46.54 & 45.3 & 33.34 & 27.06 \\
SD OB & 1.84 & 3.63 & 4.32 & 4.45 & 2.68 \\
SD NB & 1.79 & 3.85 & 4.69 & 3.26 & 2.63 \\
OB* & 0.11 & 0.44 & 0.54 & 0.61 & 0.35 \\
NB* & 0.11 & 0.55 & 0.72 & 0.48 & 0.39 \\
Difference of means & -0.231779 & 0.496497 & 0.002362 & 0.423089 & 0.465446 \\
Cl & $95 \%$ & $95 \%$ & $95 \%$ & $95 \%$ & $95 \%$ \\
IC & $(-0.557135 ; 0.093576)$ & $(-0.905768 ; 1.898762)$ & $(-1.780811 ; 1.785535)$ & $(-1.106945 ; 1.953123)$ & $(-0.577590 ; 1.508483)$ \\
T value & -1.4 & 0.7 & 0 & 0.55 & 0.89 \\
$p$ value & 0.62 & 0.484 & 0.998 & 0.584 & 0.378 \\
DF & 459 & 99 & 85 & 96 & 97 \\
\hline
\end{tabular}

*Significant values 
Table 3 Intra-group association tests (Pearson's $x^{2}$, odds ratio) of qualitative variables for the group n3 (13 to 18 years of age)

\begin{tabular}{|c|c|c|c|c|c|c|c|c|c|c|c|c|c|}
\hline Variables & $\mathrm{OBI}(\mathrm{n})$ & OBI \% NB (n) & NB \% & Total & Pearson X & $p$-value DF & Lilihood $x$ & $p$-value & DF OD & IC $95 \%$ & DF & OR & IC $95 \%$ \\
\hline $\mathrm{F}$ & 91 & 54 & 104 & 56 & & & & & & & & & \\
\hline M & 76 & 46 & 82 & 44 & & 0.072 & 0.788 & 1 & 0.072 & 0.7888 & 1 & & \\
\hline Total & 167 & & 186 & & 353 & & & & & & & & \\
\hline I & 52 & 39 & 85 & 50 & & & & & & & & & \\
\hline$\|$ & 73 & 54 & 69 & 40 & & 5.102 & $0.024^{*}$ & 1 & 5.121 & $0.024^{*}$ & 1 & 1.73 & $(1.07 ; 2.78)$ \\
\hline III & 10 & 7 & 17 & 10 & & 0.008 & 0.928 & 1 & 0.008 & 0.928 & 1 & & \\
\hline Total & 135 & & 171 & & 306 & & & & & & & & \\
\hline NMx & 21 & 13 & 15 & 8 & & & & & & & & & \\
\hline PMx & 113 & 69 & 127 & 70 & & 1.586 & 0.208 & 1 & 1.59 & 0.207 & 1 & & \\
\hline $\mathrm{RMx}$ & 29 & 18 & 40 & 22 & & 2.521 & 0.112 & 1 & 2.528 & 0.112 & 1 & & \\
\hline Total & 163 & & 182 & & 345 & & & & & & & & \\
\hline NM & 17 & 10 & 23 & 13 & & & & & & & & & \\
\hline PM & 83 & 51 & 103 & 56 & & 0.06 & 0.806 & 1 & 0.06 & 0.806 & 1 & & \\
\hline $\mathrm{RM}$ & 64 & 39 & 57 & 31 & & 1.299 & 0.254 & 1 & 1.302 & 0.254 & 1 & & \\
\hline Total & 164 & & 183 & & 347 & & & & & & & & \\
\hline NM & 45 & 28 & 63 & 35 & & & & & & & & & \\
\hline IM & 60 & 37 & 73 & 41 & & 0.288 & 0.592 & 1 & 0.288 & 0.591 & 1 & & \\
\hline SM & 56 & 35 & 42 & 24 & & 4.924 & $0.026^{*}$ & 1 & 4.943 & $0.026^{*}$ & 1 & 1.87 & $(1.07 ; 3.24)$ \\
\hline Total & 161 & & 178 & & 339 & & & & & & & & \\
\hline $\mathrm{NLAFH}$ & 27 & 17 & 47 & 27 & & & & & & & & & \\
\hline ILAFH & 109 & 68 & 103 & 58 & & 4.902 & $0.027^{*}$ & 1 & 4.957 & $0.026^{*}$ & 1 & 1.84 & $(1.07 ; 3.17)$ \\
\hline DLAFH & 25 & 15 & 26 & 15 & & & & & & & & & \\
\hline Total & 161 & & 176 & & 337 & & & & & & & & \\
\hline NP & 29 & 18 & 35 & 19 & & & & & & & & & \\
\hline $\mathrm{BP}$ & 42 & 26 & 55 & 31 & & 0.063 & 0.801 & 1 & 0.063 & 0.801 & 1 & & \\
\hline DP & 90 & 56 & 89 & 50 & & 0.465 & 0.495 & 1 & 0.466 & 0.495 & 1 & & \\
\hline Total & 161 & & 179 & & & & & & & & & & \\
\hline
\end{tabular}

*Significant $p$ values $<0.05$

OB have mandibles that are on average $2.4 \mathrm{~mm}$ wider than NB, with a $95 \%$ confidence level ( 0.4 and $4.5 \mathrm{~mm})$. Summaries of the values of the middle range are shown in Table 6.

\section{Discussion}

We assessed skeletal and dental changes in a large group of patients, to minimize the probable random errors. Statistical tests helped to widespread the results and confirmed the associations $\left(x^{2}\right)$ and disease risk (OR).

$\mathrm{OB}$ is a result of the influence of genetic and environmental factors $[4,13]$. Some authors claim that diseases such as allergies [17], chronic colds, habits [22, 27], head position [12], or shape of the upper airway [2, 7] may lead to nasal obstruction and consequent $\mathrm{OB}[1,8,12,13$, $16,28]$. However, the relative influence of these factors in the genesis of $\mathrm{OB}$ remains unclear $[2,5-7,10,11,14,20,21]$.
Studies indicate that factors that cause $\mathrm{OB}$ do not cause malocclusion [20] or modify facial patterns $[2,5]$.

Most studies included children and adolescents, because more significant changes occur in these groups $[1-10,12,16-19,21-23,27-29]$ and the changes become less apparent in adulthood [15]. Dentition and aging affect the initiation and persistence of craniofacial changes $[8,15,18]$. Alterations in early age do not persist into adulthood $[17,20]$. The influence of $\mathrm{OB}$ on craniofacial development becomes evident from the ages of 8 to 10 years [18].

Class II malocclusion remained present in all patients with $\mathrm{OB}$, as disease risk. This condition improved in adult patients in our study (n3), with only a marginal association with class II and OB $[17,20]$. It seems reasonable to suggest that early treatment of that condition may be beneficial, especially in young individuals. Treatment should be multidisciplinary [14]. 


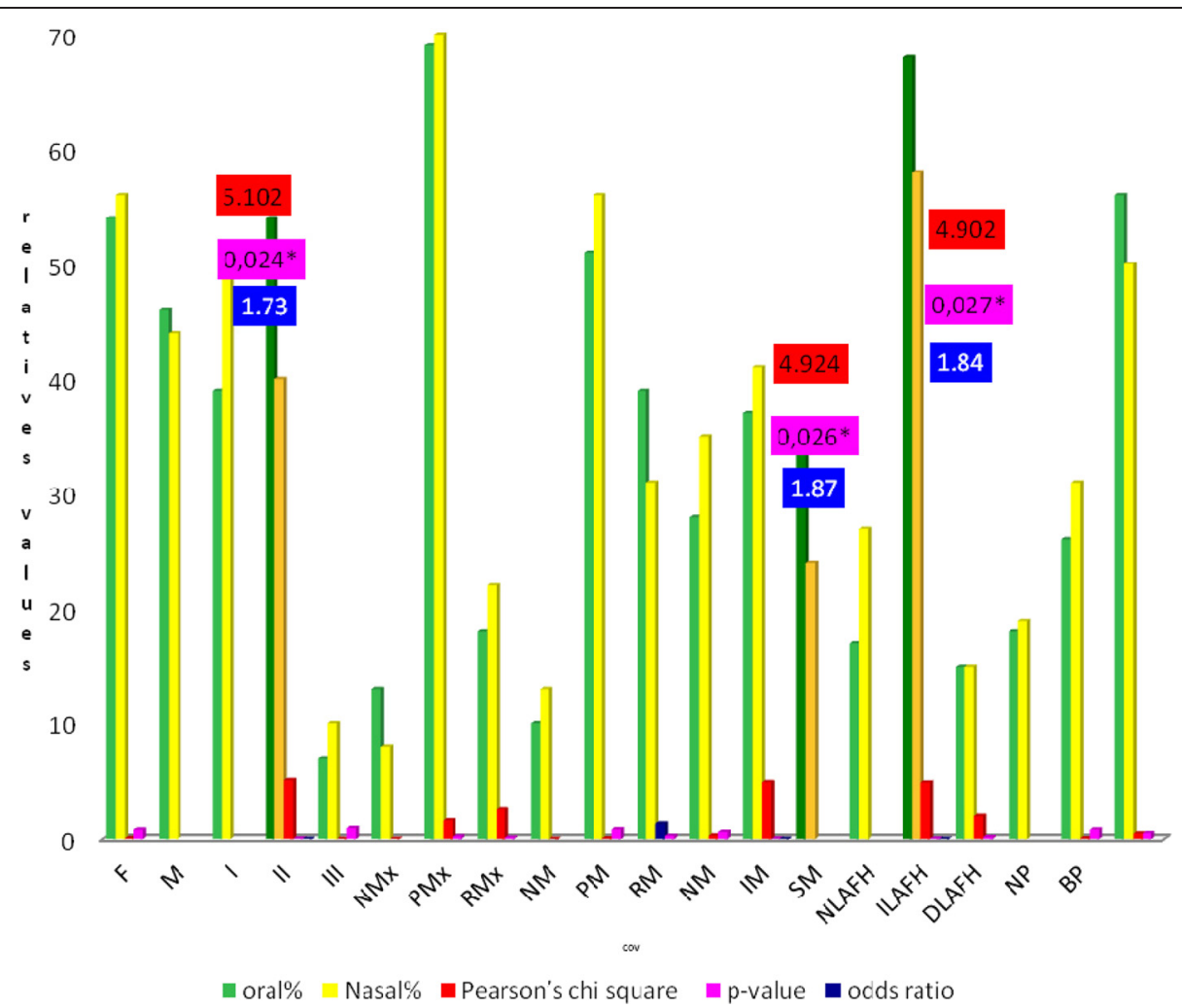

Fig. 4 Intra-groups association values (Pearson's $x^{2}$ odds ratio) for qualitative variables, n2 (924), 13 to 18 years of age, with oral and nasal breathing. *Significant values. The variables that showed significant associations and the risk of disease were Class II, SM and ILAFH

Table $4 T$ tests for difference in the averages - quantitative values for group n2 (13 to 18 years of age)

\begin{tabular}{llllll}
\hline & $(n)$ & IMSW & IMIW & ICSW & ICIW \\
\hline OB & 167 & 46 & 42 & 42 & 45 \\
NB & 186 & 49 & 46 & 45 & 45 \\
Means OB & 14.83 & 47.39 & 45.55 & 34.61 & 27.3 \\
Means NB & 15.25 & 47.02 & 46.1 & 34.5 & 27.81 \\
SD OB & 1.76 & 3.2 & 3.57 & 3.22 & 2.8 \\
SD NB & 1.7 & 3.32 & 4.22 & 3.16 & 2.9 \\
OB* & 0.14 & 0.47 & 0.55 & 0.5 & 0.31 \\
NB* & 0.12 & 0.47 & 0.62 & 0.47 & 0.43 \\
Dif of means & -0.426341 & 0.370896 & -0.550207 & 0.111905 & -0.777778 \\
Cl & $95 \%$ & $95 \%$ & $95 \%$ & $95 \%$ & $95 \%$ \\
IC & $(-0.789891 ;-0.062791)$ & $(-0.958786 ; 1.700578)$ & $(-2.203110 ; 1.102696)$ & $(-1.250651 ; 1.474461)$ & $(-1.837129 ; 0.281573)$ \\
T value & -2.31 & 0.55 & -0.66 & 0.16 & 1.46 \\
$P$ value & $0.022^{*}$ & 0.581 & 0.51 & 0.871 & 0.148 \\
DF & 343 & 92 & 85 & 84 & 79 \\
\hline
\end{tabular}

*Significant values 
Table 5 Intra-group association tests (Pearson's $x^{2}$, odds ratio) of qualitative variables - group n3 (19 to 57 years of age)

\begin{tabular}{|c|c|c|c|c|c|c|c|c|c|c|c|}
\hline Variables & $\mathrm{OB}(n)$ & OB \% & NB $(n)$ & NB \% & Total & Pearson $x^{2}$ & $p$ value & DF & Lilihood $x^{2}$ & OR & $\mathrm{Cl} 95 \%$ \\
\hline$F$ & 95 & 47 & 110 & 53 & & 1.012 & 0,314 & 1 & 1.012 & & \\
\hline M & 56 & 53 & 51 & 47 & & & & & & & \\
\hline Total & 151 & & 161 & & 312 & & & & & & \\
\hline | & 36 & 32 & 57 & 43 & & & & & & & \\
\hline$\|$ & 63 & 56 & 58 & 43 & & 3.773 & $0.052^{*}$ & 1 & 3.792 & 1.72 & $(0.99 ; 2.98)$ \\
\hline III & 14 & 12 & 19 & 14 & & 0.14 & 0.708 & 1 & 0.14 & & \\
\hline Total & 113 & & 134 & & 247 & & & & & & \\
\hline NMx & 15 & 10 & 8 & 5 & & & & & & & \\
\hline PMx & 107 & 73 & 122 & 80 & & 2.862 & 0.091 & 1 & 2.894 & & \\
\hline RMx & 25 & 17 & 22 & 15 & & 0.912 & 0.34 & 1 & 0.923 & & \\
\hline Total & 147 & & 152 & & 299 & & & & & & \\
\hline NM & 21 & 14 & 16 & 10 & & & & & & & \\
\hline PM & 81 & 55 & 90 & 59 & & 1.073 & 0.3 & 1 & 1.075 & & \\
\hline RM & 46 & 31 & 47 & 31 & & 0.564 & 0.453 & 1 & 0.565 & & \\
\hline Total & 148 & & 153 & & 301 & & & & & & \\
\hline NM & 42 & 30 & 39 & 26 & & & & & & & \\
\hline IM & 57 & 40 & 61 & 41 & & 0.242 & 0.623 & 1 & 0.242 & & \\
\hline SM & 43 & 30 & 48 & 33 & & 0.363 & 0.547 & 1 & 0.363 & & \\
\hline Total & 142 & & 148 & & 290 & & & & & & \\
\hline NLAFH & 26 & 18 & 34 & 23 & & & & & & & \\
\hline ILAFH & 100 & 71 & 94 & 63 & & 1.237 & 0.266 & 1 & 1.24 & & \\
\hline DLAFH & 15 & 11 & 21 & 14 & & 0.026 & 0.873 & 1 & 0.026 & & \\
\hline Total & 141 & & 149 & & 290 & & & & & & \\
\hline NP & 22 & 16 & 26 & 18 & & & & & & & \\
\hline $\mathrm{BP}$ & 43 & 31 & 50 & 34 & & 0.002 & 0.964 & 1 & 0.002 & & \\
\hline DP & 74 & 53 & 70 & 48 & & 0.444 & 0.505 & 1 & 0.445 & & \\
\hline Total & 139 & & 146 & & 285 & & & & & & \\
\hline
\end{tabular}

*Significant values

In our groups, we observed a higher proportion of subjects class II. Initially, we made the separation of groups by age and by mode of breathing, so the occlusal and skeletal variables were divided into groups, regardless of their initial ratio.

We obtained different results for each age group. In group $\mathrm{n} 1$, most children BP and with DLAFH, breathe through the nose and were class I, and these characteristics were shown to be protective factors found in the OR [18]. In group n2, we found that the oral breathers tended to be younger than nasal breathers. We did not identify sexual dimorphism in any group [18], although some studies claim a slight prevalence of $\mathrm{OB}$ in females $[5,13]$. There were fewer changes in group $\mathrm{n} 3$ that exhibited only significant associations with dental changes as class II malocclusion and mandibular width suggesting an increased tendency to posterior cross bite, agreeing with many studies $[2,5,10,15-17,19,20]$ and disagreeing with others $[1,3]$. Most patients reported "crooked teeth" as the main complain, and the information of $\mathrm{OB}$ and $\mathrm{NB}$ was obtained following the protocol $[1,2,6$, $8-11,13,19,28]$.

Some authors postulate that facial, skeletal $[1,7,8,10,13$, $15,17,20,28]$, and dental $[1,7,8,15,17,20,22,28]$ changes result from the influence of oral breathing $[1,7,13,29]$. We confirmed this statement: In group 1, we found evidence of associations of $\mathrm{SM}$ and $\mathrm{RM}$ and $\mathrm{OB}$ in agreement with several studies $[8,10,13,15,28]$, and a DLAFH and BP with $\mathrm{NB}$, which appeared to be protective for disease, in disagreement with some studies $[8,10,13,15,28]$. In group n2 we found a clear association of SM and ILAFH agreeing with previous publications $[2,10,16]$. In group 3 , we found a marginally significant association between class II malocclusion and $\mathrm{OB}$, confirming the literature $[8,15,18]$.

The condition of exclusive oral breathing is rare; alternation between $\mathrm{OB}$ and $\mathrm{NB}$ is much more common. The boundary between the air passages for $\mathrm{OB}$ and NB is small, 


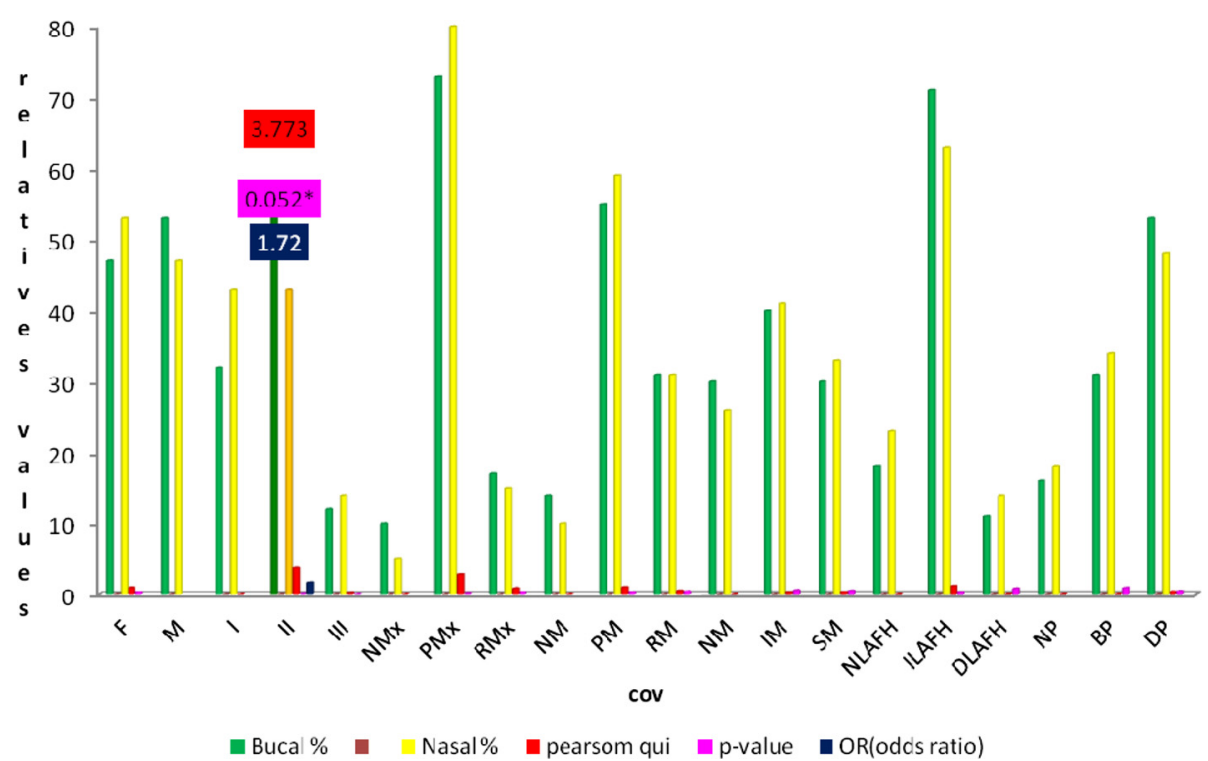

Fig. 5 Intra-groups association values, (Pearson's $x^{2}$, OR, for qualitative variables n3 (19 to 57 years of age), with OB and NB. *Significant values. The variable that showed significant associations and the risk of disease was the Angle Class II

and sometimes, even though the nasal air passages are of normal size, nasal breathing is replaced by oral $[3,16]$.

We could not obtain concordances in the literature for the diagnosis of OB. Few studies evaluated OB using calibrated instruments, but they are not gold standards. Even as the assessments may have been made at a time when the breathing mode was alternating $[3,12,16,23]$.

Most of the literature uses subjective information obtained through questionnaires, where the patient states his mode of breathing, and some clinical trials are not carefully constructed and do not lead to results that generate confidence $[1,2,8-11,13,19,20,28]$. Our diagnosis of $\mathrm{OB}$ and $\mathrm{NB}$ was based on clinical examination and a questionnaire [11].

\section{Conclusions}

This study showed that dental and skeletal factors are associated with $\mathrm{OB}$ in children, and it seems that it becomes more severe until adolescence. But adults showed no associations between $\mathrm{OB}$ and skeletal factors, only in dental variables, indicating that there is no cause-effect relationship between the dental and skeletal factors and

Table $6 T$ test for differences of averages- Quantitative values for group n3 (19 to 57 years of age)

\begin{tabular}{llllll}
\hline & $(n)$ & IMSW & IMIW & ICSW & ICIW \\
\hline OB & 151 & 41 & 36 & 43 & 43 \\
NB & 161 & 43 & 39 & 37 & 37 \\
Means OB & 26.17 & 47.93 & 47.07 & 35.01 & 28.37 \\
Means NB & 27.95 & 46.7 & 44.65 & 34.86 & 27.64 \\
SD OB & 6.71 & 4.01 & 4.09 & 4.57 & 3.03 \\
SD NB & 7.35 & 4.29 & 4.74 & 3.61 & 2.91 \\
OB* & 0.55 & 0.63 & 0.68 & 0.7 & 0.46 \\
NB* & 0.58 & 0.65 & 0.66 & 0.79 & 0.48 \\
Dif of means & -1.77813 & 1.22915 & 2.4156 & 0.146763 & 0.736958 \\
Cl & $95 \%$ & $95 \%$ & $95 \%$ & $95 \%$ & $95 \%$ \\
IC & $(-3.34477 ;-0.21148)$ & $(-0.57221 ; 3.03052)$ & $(0.38313 ; 4.44807)$ & $(-1.675761 ; 1.969287)$ & $(-0.587233 ; 2.061149)$ \\
T value & -2.23 & 1.36 & 2.37 & 0.16 & 1.11 \\
$p$ value & $0.026^{*}$ & 0.178 & $0.027^{*}$ & 0.873 & 0.271 \\
DF & 309 & 81 & 72 & 77 & 77 \\
\hline
\end{tabular}

*Significant

There was a significant difference between the $\mathrm{OB}$ and $\mathrm{NB}: \mathrm{OB}$ are younger, and the inferior molar widths are larger than the superior molar widths 
OB. The treatment of nose breathing patient should be multidisciplinary, since $\mathrm{OB}$ remains even when dental and skeletal factors slow down.

\begin{abstract}
Abbreviations
BP: brachycephalic facial pattern; CG: control group; Cl: confidence interval; DLAFH: decreased lower anterior facial height; DP: dolichocephalic pattern; IC: intercanine; ICIW: intercanine width inf; ICSW: intercanine width sup; ILAFH: increased lower anterior facial height; IMIW: intermolar width inf; IMSW: intermolar width sup; LM: large mandible; NB: nasal breathing; NLAFH: normal lower anterior facial height; NM: normal mandible; NP: normocephalic pattern; OB: oral breathing; OR: odds ratio; OSA: sleep apnea; PM: protruded mandible; PMx: protruded maxilla; RM: retruded mandible; RMx: retruded maxilla; SD: standard deviations; SG: study group; SM: short mandible.
\end{abstract}

\section{Competing interests}

The authors declare that they have no competing interests.

\section{Authors' contributions}

RRC participated in all phases of the study. RNJ and RNC participated in data collection. YHK collaborated with images evaluation and measurements. PSSn coordinated the study and collaborated with writing and reviewing of the text. All authors read and approved the final manuscript.

\section{Acknowledgements}

This clinical study was conducted at the Federal University of Sao Paulo (UNIFESP), and it is part of the thesis for obtaining the title of Doctor DDs, Ms, Rosa Carrieri Rossi. There was no previous publication of this research. The Institutional Ethics Committee (UNIFESP) approved the study project (1739/11 02/12/2011).

\section{Financial support}

The research was financially supported by Fundação Capes Ministério de Educação-Brasil CAPES Foundation-MEC Brasil. The authors, the educational institutions and the agency responsible for Research, have no conflict of interest.

\section{Author details}

'Division of Pediatric Otolaryngology, Federal University of Sao PauloUNIFESP Brasil, Rua Botucatu 740, 4 andar, V. Clementino, São Paulo CEP:04023-062, Brazil. ${ }^{2}$ Educational Association of Brazil- FUNORTE/SOEBRAS, Rua Tijuco Preto 1694, Sao Paulo, Tatuapé CEP:03316-000, Brazil. 'Department of Diagnostic Imaging, Division of Otolaryngology and Head and Neck Imaging, Federal University of Sao Paulo- UNIFESP, Rua Botucatu 740, 4 andar, V. Clementino, São Paulo CEP:04023-062, Brazil. ${ }^{4}$ Department of Otolaryngology and Head and Neck Surgery, Division of Pediatric Otolaryngology, Federal University of Sao Paulo- UNIFESP, Rua Botucatu 740, 4 andar, V. Clementino, São Paulo CEP:04023-062, Brazil.

Received: 19 February 2015 Accepted: 13 June 2015

Published online: 15 July 2015

\section{References}

1. Kluemper GT, Vig PS, Vig KW. Nasorespiratory characteristics and craniofacial morphology. The European Journal of Orthodontics. 1995;17(6):491-5.

2. Bianchini AP, Guedes ZC, Vieira MM. A study on the relationship between mouth breathing and facial morphological pattern. Braz J Otorhinolaryngol. 2007;73(4):500-5.

3. Warren DW, Hairfield WM, Seaton D, Morr KE, Smith LR. The relationship between nasal airway size and nasal-oral breathing. Am J Orthod Dentofacial Orthop. 1988;93(4):289-93.

4. Retamoso LB, Knop LAH, Guariza Filho O, Tanaka OM. Facial and dental alterations according to the breathing pattern. J Appl Oral Sci. 2011;19(2):175-81.

5. Coelho ARDP, Tanaka O, Ribeiro JS, Machado MAN, Camargo ES. Transverse craniofacial dimensions in Angle Class II, Division 1 malocclusion according to breathing mode Braz. Oral Res. 2010;24(1):70-5.

6. de OLIVEIRA JML, Carvalho ES, Dutra ALT, Bezerra ACB, de TOLEDO OA. Transverse measurements of the jaws of patients with a predominance of nose and mouth breathing in children between 6 and 14 years of age. RGO. 2012;60(1):61-9.

7. Ucar Fl, Uysal T. Comparision of orofacial airway dimensions in subject with different breathing pattern. Progress in Orthod. 2012;13(3):210-7.

8. Harari D, Redlich M, Miri S, Hamud T, Gross M. The effect of mouth breathing versus $\mathrm{OB}$ on dentofacial and craniofacial development in orthodontic patients. Laryngoscope. 2010;120(10):2089-93.

9. Bakor SF, Enlow DH, Pontes P, De Biase NG. Craniofacial growth variations in nasal-breathing, oral-breathing, and tracheotomized children. Am J Orthod Dentofacial Orthop. 2011;140(4):486-92.

10. Malhotra S, Pandey RK, Nagar A, Agarwal SP, Gupta VK. The effect of mouth breathing on dentofacial morphology of growing child. J Indian Soc Pedod Prev Dent. 2012;30:27-31.

11. Wieler WJ, Barros AM, Barros LA, Camargo ES, Ignácio SA, Maruo H. A combined protocol to aid diagnosis of breathing mode. Rev Clin Pesq Odontol. 2007;3(2):101-14.

12. Solow B, Siersbæk-Nielsen S, Greve E. Airway adequacy, head posture, and craniofacial morphology. Am J Orthod. 1984;86(3):214-23.

13. Padzys GS, Tankosic C, Trabalon M, Martrette JM. Craniofacial development and physiological state after early oral breathing in rats. Eur J Oral Sci. 2012;120(1):21-8.

14. Harvold EP, Tomer BS, Vargervik K, Chierici G. Primate experiments on oral respiration. Am J Orthod. 1981;79(4):359-72.

15. Souki BQ, Pimenta GB, Souki MQ, Franco LP, Becker HM, Pinto JA. Prevalence of malocclusion among mouth breathing children: do expectations meet reality? Int J Pediatr Otorhinolaryngol. 2009;73:767-73.

16. Zicari AM, Albani F, Ntrekou P, Rugiano A, Duse M, Mattei A, et al. Oral breathing and dental malocclusions. European journal of paediatric dentistry: official journal of European Academy of Paediatric Dentistry. 2009;10(2):59-64.

17. Bresolin D, Shapiro PA, Shapiro GG, Chapko MK, Dassel S. Mouth breathing in allergic children: its relationship to dentofacial development. Am J Orthod. 1983:83(4):334-40.

18. Muñoz ICL, Orta PB. Comparison of cephalometric patterns in mouth breathing and nose breathing children. Int J Pediatr Otorhinolaryngol. 2014;78(7):1167-72.

19. Mattar SEM, Matsumoto MAN, Valera FCP, Anselmo-Lima WT, Faria G. The effect of adenoidectomy or adenotonsillectomy on occlusal features in mouth-breathing. Pediatr Dent. 2012;34(5):108-12.

20. Souk BQ, Petrus B, Lopes TBJ, Pereira LP, Franco HMG, Becker DO. Mouth breathing children and cephalometric pattern: does the stage of dental development matter? Int J Pediatr Otorhinolaryngol. 2012;76:837-8.

21. Young T, Larel F, Hyon K. Nasal obstruction as a risk factor for sleep-disordered breathing. J Allergy Clin Immunol. 1997;99(2):s757-62.

22. Vig PS, Sarver DM, Hall DJ, Warren DW. Quantitative evaluation of nasal airflow in relation to facial morphology. Am J Orthod. 1981;79(3):263-72.

23. Fujimoto S, Yamaguchi K, Gunjigake K. Clinical estimation of mouth breathing. Am J Orthod Dentofacial Orthop. 2009;136(5):e630-1.

24. Namara Jr M. A method for cephalometric evaluation. Am J Orthod. 1984;86(5):449-69.

25. Durão AR, Pittayapat P, Rockenbach MI, Olszewski R, Ferreira AP, Jacobs R. Validity of 2D lateral cephalometry in orthodontics: a systematic review. Progress in Orthod. 2013;14:31.

26. Angle EH. Classification of malocclusion. Dental Cosmos 1899: 248-264.

27. MCNAMARA JA. Influence of respiratory pattern on craniofacial growth. Angle Orthod. 1981;51(4):269-300.

28. Trask GM, Shapiro GG, Shapiro PA. The effects of perennial allergic rhinitis on dental and skeletal development: a comparison of sibling pairs. Am J Orthod Dentofacial Orthop. 1987;92(4):286-93.

29. Souki BQ, Lopes PB, Veloso NC, Avelino RA, Pereira TB, Souza PE, et al. Facial soft tissues of mouth-breathing children: do expectations meet reality? Int J Pediatr Otorhinolaryngol. 2014;78(7):1074-9. 